\title{
Energy Levels and Radiative Rates in Ge(XVIII)
}

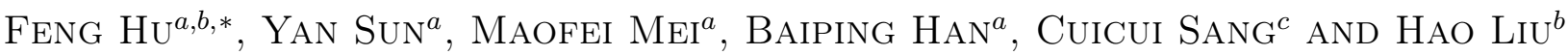 \\ ${ }^{a}$ School of Mathematical and Physical Sciences, Xuzhou University of Technology, \\ Xuzhou 221018, People's Republic of China \\ ${ }^{b}$ Research Center of Laser Fusion, China Academy of Engineering Physics, \\ Mianyang 621900, People's Republic of China \\ ${ }^{c}$ School of Sciences, Lanzhou University of Technology, Lanzhou 730050, People's Republic of China
}

(Received December 12, 2019; in final form March 4, 2020)

Energy levels and E1, E2, M1, and M2 transitions rates are reported for the low-lying 41 levels of Ge(XVIII), belonging to the $n=3$ states $\left(1 s^{2} 2 s^{2} 2 p^{6}\right) 3 s^{2} 3 p^{3}, 3 s 3 p^{4}$, and $3 s^{2} 3 p^{2} 3 d$. High-accuracy calculations have been performed as benchmarks for accurate treatments of relativity, electron correlations, and QED effects in multivalence-electron systems. The calculated energy levels are in excellent agreement with the experimental results and the experimentally compiled energy values from the National Institute for Standards and Technology wherever available. The calculated values, including core-valence corrections, are found to be in a good agreement with other theoretical and experimental values. The present results are reported as benchmarks for future calculations and measurements.

DOI: 10.12693/APhysPolA.137.1141

PACS/topics: energy levels, radiative rates, transition probabilities

\section{Introduction}

The opacity is an important issue in the knowledge of the radiative properties of inertial confinement fusion (ICF) and astrophysical plasmas. The opacity of germanium dopant embedded in the ablator of some ICF capsules was presented in the recent years [1]. Electron temperature and density measurements of laser produced germanium plasma were observed by Shakeel et al. [2] The X-ray emission spectra of germanium plasma were produced by Comet et al. [3]. The parameters such as temperature and density were deduced from hydrodynamic simulations and collisional-radiative computations by using the opacity codes [3]. The opacity code provided a comprehensive discussion of the rate and energy level calculations involved in producing a detailed term accounting (DTA) model within a consistent framework [4]. A DTA model needs the accurate results of energy levels, transition probabilities, oscillator strengths, and lifetimes for each state of ions [5-7].

For Ge(XVIII) ion, only few studies have been were made. The magnetic-dipole lines for Ge(XVIII) were given by Sugar and Kaufman with the configuration interaction [8]. Theoretical energy levels and transition probabilities were presented for Ge(XVIII) by Huang with the multiconfiguration Dirac-Fock (MCDF) technique [9]. Transition probabilities for forbidden lines in the ground configuration of phosphorus sequence from $\mathrm{Ar}$ to $\mathrm{Ru}$ were reported by Biemont with the configuration interaction [10]. E2 and M1 transitions with the nuclear charge in the range of $28 \leq Z \leq 42$ were given by Froese Fischer using the multiconfiguration Hartree-Fock

\footnotetext{
* corresponding author
}

method and Breit-Pauli approximation [11]. The M1 line strengths among the levels of the $3 s^{2} 3 p^{3}$ ground configuration of the $\mathrm{P}$ sequence were plotted by Curtis et al. with the empirical determination [12]. Transition energies and probabilities as well as oscillator strengths for E1 transitions to the $3 s^{2} 3 p^{3}$ ground configuration were tabulated by Fritzsche et al. with the MCDF method [13]. Oscillator strengths for the dipole-allowed $3 p^{3}{ }^{4} S^{o}-3 p^{2}\left({ }^{3} P\right) 3 d^{4} P$ transitions were performed by Charro et al. with the relativistic quantum defect orbital (RQDO) method [14]. Term energies of four configurations in ions of the phosphorus isoelectronic sequence $(Z=26-32)$ were evaluated by Vilkas and Ishikawa with the relativistic multireference many-body perturbation (MR-MP) theory [15]. E1 - forbidden transition rates for P-like ions were summarized by Träbert [16-17].

Träbert conducted a critical assessment of theoretical calculations of structure and transition probabilities from an experimenter's view [18]. He pointed out that new computations can match measurement, fill gaps, and suggest revisions closely with almost spectroscopic accuracy. Moreover, these citations of theoretical works as well as the ones for experimental data are certainly incomplete. These citations were a number of studies of P-like ions calculations where the attention was paid to the trend. So, limited energy levels or transitions were considered, or some selected configurations were discussed [8-17]. Some results were given in the form of graphs [12]. There are still some existing problems such as the identification of terms and strong mixing of configurations, which will be discussed in detail in the next part of this paper.

In this paper, the MCDHF method is performed to calculate the E1, E2, M1, and M2 wavelengths, oscillator strengths, transition probabilities, and fine-structure levels for Ge(XVIII) using the new release of the GRASP2K code $[19,20]$. Configurations $\left(1 s^{2} 2 s^{2} 2 p^{6}\right) 3 s^{2} 3 p^{3}, 3 s 3 p^{4}$, 
and $3 s^{2} 3 p^{2} 3 d$ are reported in this calculation. Based on our previous work [21, 22], in this paper, the valencevalence $(\mathrm{VV})$ and core--valence $(\mathrm{CV})$ correlation effects are considered in a systematic way. The Breit interactions and QED effects have been added. This computational approach enables us to present a consistent and improved data set of all important E1, E2, M1, and M2 transitions of the Ge(XVIII) spectra, which are useful for identifying transition lines in further investigations.

\section{Method}

\subsection{Computational procedure}

The MCDHF method has recently been described in great detail by Grant [23]. Hence, we only repeat the essential features here. Starting from the Dirac-Coulomb Hamiltonian

$$
H_{\mathrm{DC}}=\sum_{i=1}^{N}\left(c \alpha_{i} \cdot \boldsymbol{p}_{i}+\left(\beta_{i}-1\right) c^{2}+V_{i}^{N}\right)+\sum_{i>j}^{N} \frac{1}{r_{i j}},
$$

where $V^{N}$ is the monopole part of the electron-nucleus Coulomb interaction, the atomic state functions (ASFs) describing different fine-structure states are obtained as linear combinations of symmetry adapted configuration state functions (CSFs):

$$
\left|\gamma J M_{J}\right\rangle=\sum_{j=1}^{N_{C S F s}} c_{j}\left|\gamma_{j} J M_{J}\right\rangle
$$

In the expression above $J$ and $M_{J}$ are the angular quantum numbers. $\gamma$ denotes other appropriate labeling of the configuration state function, for example parity, orbital occupancy, and coupling scheme. The configuration state functions are built from products of one-electron Dirac orbitals. In the relativistic self-consistent field (RSCF) procedure both the radial parts of the Dirac orbitals and the expansion coefficients are optimized to selfconsistency. The Breit interaction

$$
\begin{aligned}
& H_{\text {Breit }}=-\sum_{i<j}^{N}\left[\alpha_{i} \cdot \alpha_{j} \frac{\cos \left(\omega_{i j} r_{i j} / c\right)}{r_{i j}}\right. \\
& \left.+\left(\alpha_{i} \cdot \nabla_{i}\right)\left(\alpha_{j} \cdot \nabla_{j}\right) \frac{\cos \left(\omega_{i j} r_{i j} / c\right)-1}{\omega_{i j}^{2} r_{i j} / c^{2}}\right],
\end{aligned}
$$

as well as leading QED corrections can be included in subsequent relativistic configuration interaction (RCI) calculations [24]. Calculations cannot only be done for single levels, but also for portions of a spectrum in the extended optimal level (EOL) scheme, where optimization is on a weighted sum of energies [25]. Using the latter scheme, a balanced description of a number of finestructure states belonging to one or more configurations can be obtained in a single calculation.

\subsection{Generation of configuration expansions}

In this work, we included different correlations into the calculation in a systematic approach. The correlation energy is defined as the energy difference between the exact solution to the Dirac equation and the DF solution. The contribution from different types of correlations then can be defined as the energy difference between the solution including the particular correlation under investigation and the DF solution. To classify the correlation, the atomic electrons can be divided into two parts: valence electrons and core electrons. As a result, the correlation between the valence electrons is defined as VV correlation, and the correlation between the valence electrons and core electrons is defined as CV correlation.

It is, from some perspectives, desirable to perform separate calculations for each of the studied atomic states. This approach, however, is impractical and time consuming. Instead, the atomic state functions for a number of closely spaced levels were determined together in the socalled EOL procedure [25]. For the calculations of lowlying levels of $\mathrm{P}$-like ions, the $3 s^{2} 3 p^{3}$ configuration with total angular momenta $J=1 / 2,3 / 2$, and $5 / 2$, the $3 s 3 p^{4}$ configurations with total angular momenta $1 / 2,3 / 2$, and $5 / 2$, and the $3 s^{2} 3 p^{2} 3 d$ configuration with total angular momenta $J=1 / 2,3 / 2,5 / 2,7 / 2$, and $9 / 2$ were used.

In the MCDHF approach, the correlation is represented by different constraints on the generation of the CSFs included in Eq. (2). If we only include the VV, the core electrons are kept fixed in all the CSFs generated. To include CV, we allow one of the core electrons to be excited to generate the CSFs.

\subsection{Calculation procedure}

The $3 s^{2} 3 p^{3}, 3 s 3 p^{4}$, and $3 s^{2} 3 p^{2} 3 d$ configurations define the multireference (MR) for the even and odd parities, respectively. As a starting point, MCDHF calculations in the EOL scheme were performed for even and odd states using configuration expansions including all lower states of the same $J$ symmetry and parity. A Dirac-Coulomb version was used for the optimization of the orbitals, including the Breit corrections in a final configuration interaction calculation [23].

The calculations for the even states were based on CSF expansions obtained by allowing single $(\mathrm{S})$ and double (D) substitutions of orbitals in the even MR configurations to an increasing active set of orbitals. In a similar way the calculations for the odd states were based on CSF expansions obtained by allowing single $(\mathrm{S})$ and double (D) substitutions of orbitals in the odd MR configurations to an increasing active set of orbitals. Larger orbital sets can result in a considerable increase of computational time required for the problem, and appropriate restrictions may be necessary. We divided up the calculations into two parts, one where we optimized a set of orbitals for the even states and one for the odd states, i.e. the upper and lower states were described by two independently optimized sets of orbitals. Because of this we had to use biorthogonal transformation [26] of the atomic state functions to calculate the transition parameters. 
The similar calculation procedure have been introduced in Ref. [21]. Here, we only give an outline. For $\mathrm{P}$-like ions, the ground and first excited configurations are $3 s^{2} 3 p^{3}$ and $3 s^{2} 3 p^{2} 3 d$ respectively. In the first step, the active set (AS) is

$$
\mathrm{AS} 1=\{3 s, 3 p, 3 d\} .
$$

Then, we increase the active set in the way shown as follows:

$$
\begin{aligned}
& \mathrm{AS} 2=\mathrm{AS} 1+\{4 s, 4 p, 4 d, 4 f\}, \\
& \mathrm{AS} 3=\mathrm{AS} 2+\{5 s, 5 p, 5 d, 5 f, 5 g\}, \\
& \mathrm{AS} 4=\mathrm{AS} 3+\{6 s, 6 p, 6 d, 6 f, 6 g\}, \\
& \mathrm{AS} 5=\mathrm{AS} 4+\{7 s, 7 p, 7 d, 7 f, 7 g\} .
\end{aligned}
$$

Different active sets are used in VV and CV. Here, we discuss each clearly.

In $\mathrm{VV}$ method, we set $1 s^{2} 2 s^{2} 2 p^{6}$ as core electrons in the calculation. Then we increase the principal quantum number $n$, and optimize the orbitals AS1, AS2, AS3, AS4, and AS5.

In CV model, we set $1 s^{2} 2 s^{2} 2 p^{5}$ as core electrons. Then we optimize the layer by $n$. We generate the CSFs of the form of $1 s^{2} 2 s^{2} 2 p^{5} \mathrm{AS} n, n=1-4$. Also, the CSFs of CV have the form of $1 s^{2} 2 s^{1} 2 p^{6} \mathrm{AS} n, n=1-4$.

\section{Results and discussion}

The success of a calculation relies on a judiciously chosen configuration expansion [27]. To ensure the convergence of a calculated expectation value within a certain correlation model, the configuration expansion must be enlarged in a systematic way. A very efficient way of doing this is to use the active set method, where $j j$-coupled CSFs of a specified parity $P$ and angular momentum $J$ symmetry are generated by excitations from one or more reference configurations to an active set of orbitals. The convergence of the atomic property can then be studied as a function of the size of the active set. The GRASP2K procedure JJ2LSJ [28] was used for the transformation of ASFs from a $j j$-coupled CSF basis into an $L S J$-coupled CSF basis [29] for the results.

Results for $\left(1 s^{2} 2 s^{2} 2 p^{6}\right) 3 s^{2} 3 p^{3}, 3 s 3 p^{4}$, and $3 s^{2} 3 p^{2} 3 d$ configurations of Ge(XVIII) give rise to the low-lying 41 levels listed in Table I, where we compare our results with the experimental data compiled by the National Institute for Standards and Technology (NIST) [30]. The NIST database lists the energies for the 11 out of the present 41 excited-levels in Ge(XVIII). The principal number was limited to $n=7$. There are two reasons for this. One is the convergence as mentioned above. For VV calculation, it is not very difficult to get convergence for higher principal number $(n \geq 8)$, but for $\mathrm{CV}$ calculation the convergence is difficult. The number of CSFs would increase very rapidly when we include the $n \geq 8$ orbitals, and it is hard to get convergence. Also, due to the

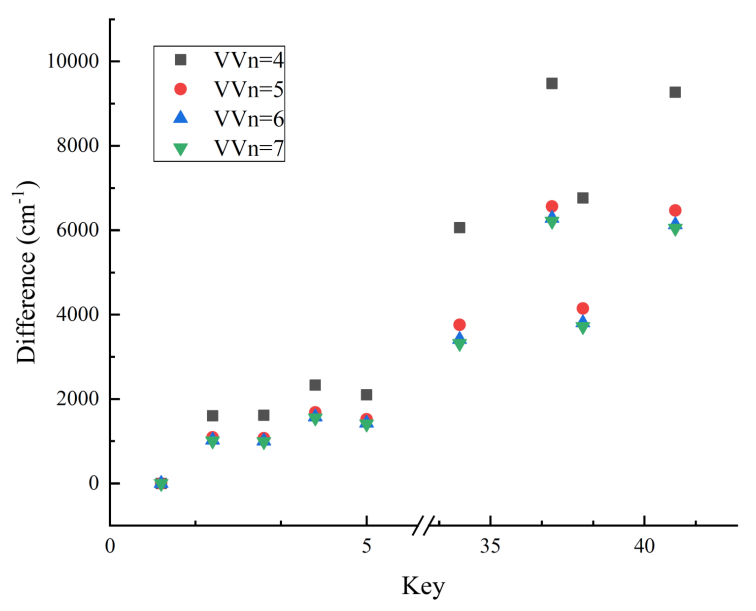

Fig. 1. Energy difference between the valence-valence correlation results and the energies for the 11 out of the lowest 41 levels from NIST.

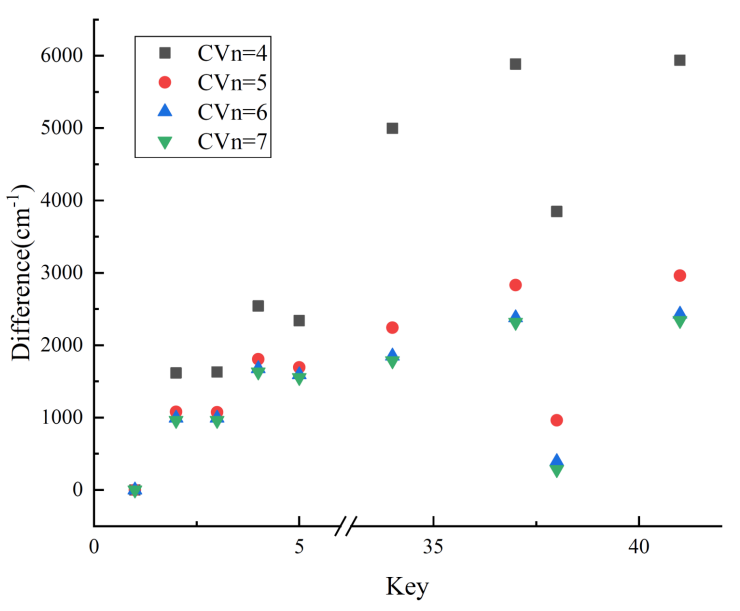

Fig. 2. As in Fig. 1, but for the core-valence correlation results.

computational calculation limit and the problem of the program GRASP2K code itself, we only compare the VV and CV models on an equal footing $(n \leq 7)$, as mentioned above. The other is the fact that contribution from $n=7$ is less than $0.01 \%$.

Figure 1 shows the mean (with the standard deviation) of the relative differences between $\mathrm{VV}_{n}$ and NIST which are 1239.5 and $4727.5 \mathrm{~cm}^{-1}$, respectively. The smallest difference is $990 \mathrm{~cm}^{-1}$ lower than NIST $\left(3 s^{2} 3 p^{3}\left({ }_{3}^{2} D\right)^{2} D_{5 / 2}\right)$, and the biggest difference can be up to $9270 \mathrm{~cm}^{-1}\left(3 s^{2} 3 p^{2}\left({ }_{2}^{1} D\right)^{1} D 3 d^{2} D_{5 / 2}\right)$.

Figure 2 shows the mean (with the standard deviation) of the relative differences between $\mathrm{CV}_{n}$ and NIST which are 1275.5 and $1417.5 \mathrm{~cm}^{-1}$, respectively. This can be treated as a good example of calculations with the necessary correlations included. As can be seen from Fig. 1 and Fig. 2, some results considering more configurations are not better than those with fewer configurations. This can be due to configuration mixing. 
Energies (in $\mathrm{cm}^{-1}$ ) relative to the ground state for the lowest 41 levels in Ge(XVIII).

\begin{tabular}{|c|c|c|c|c|c|c|c|c|c|c|}
\hline Key & Configuration & $\mathrm{VV}_{n=4}$ & $\mathrm{VV}_{n=5}$ & $\mathrm{VV}_{n=6}$ & $\mathrm{VV}_{n=7}$ & $\mathrm{CV}_{n=4}$ & $\mathrm{CV}_{n=5}$ & $\mathrm{CV}_{n=6}$ & $\mathrm{CV}_{n=7}$ & Exp. \\
\hline 1 & $3 s^{2} 3 p^{3}\left({ }_{3}^{4} S\right)^{4} S_{3 / 2}$ & 0 & 0 & 0 & 0 & 0 & 0 & 0 & 0 & 0 \\
\hline 2 & $3 s^{2} 3 p^{3}\left({ }_{3}^{2} D\right)^{2} D_{3 / 2}$ & 57720 & 57210 & 57141 & 57126 & 57733 & 57197 & 57109 & 57077 & 56117 \\
\hline 3 & $3 s^{2} 3 p^{3}\left({ }_{3}^{2} D\right)^{2} D_{5 / 2}$ & 75260 & 74717 & 74650 & 74636 & 75276 & 74719 & 74637 & 74606 & 73646 \\
\hline 4 & $3 s^{2} 3 p^{3}\left({ }_{1}^{2} P\right)^{2} P_{1 / 2}$ & 114661 & 114018 & 113909 & 113885 & 114875 & 114140 & 114010 & 113963 & 112334 \\
\hline 5 & $3 s^{2} 3 p^{3}\left({ }_{1}^{2} P\right)^{2} P_{3 / 2}$ & 144231 & 143652 & 143559 & 143539 & 144471 & 143826 & 143724 & 143685 & 142132 \\
\hline 6 & $3 s^{2} S 3 p^{4}\left({ }_{2}^{3} P\right)^{4} P_{5 / 2}$ & 406992 & 406968 & 406889 & 406869 & 407827 & 407230 & 407283 & 407243 & \\
\hline 7 & $3 s^{2} S 3 p^{4}\left({ }_{2}^{3} P\right)^{4} P_{3 / 2}$ & 436100 & 436101 & 436027 & 436008 & 437008 & 436440 & 436507 & 436471 & \\
\hline 8 & $3 s^{2} S 3 p^{4}\left({ }_{2}^{3} P\right)^{4} P_{1 / 2}$ & 445559 & 445537 & 445454 & 445433 & 446492 & 445888 & 445949 & 445910 & \\
\hline 9 & $3 s^{2} S 3 p^{4}\left({ }_{2}^{1} D\right)^{2} D_{3 / 2}$ & 506864 & 506508 & 506340 & 506293 & 507212 & 506279 & 506241 & 506181 & \\
\hline 10 & $3 s^{2} S 3 p^{4}\left({ }_{2}^{1} D\right)^{2} D_{5 / 2}$ & 517285 & 516932 & 516767 & 516720 & 517751 & 516796 & 516764 & 516704 & \\
\hline 11 & $3 s^{2} 3 p^{2}\left({ }_{2}^{3} P\right)^{3} P 3 d^{2} P_{3 / 2}$ & 575286 & 574935 & 574768 & 574723 & 574682 & 573901 & 573834 & 573802 & \\
\hline 12 & $\left.3 s^{2} 3 p^{2}{ }_{2}^{3} P\right)^{3} P 3 d^{2} P_{1 / 2}$ & 582319 & 581733 & 581489 & 581426 & 581814 & 580633 & 580468 & 580386 & \\
\hline 13 & $3 s^{2} 3 p^{2}\left({ }_{2}^{3} P\right)^{3} P 3 d^{4} F_{3 / 2}$ & 603871 & 603200 & 603055 & 603021 & 603306 & 602259 & 602193 & 602207 & \\
\hline 14 & $3 s^{2} 3 p^{2}\left({ }_{2}^{3} P\right)^{3} P 3 d^{4} F_{5 / 2}$ & 615733 & 615088 & 614949 & 614918 & 615214 & 614200 & 614152 & 614173 & \\
\hline 15 & $3 s^{2} S 3 p^{4}\left({ }_{0}^{1} S\right)^{2} S_{1 / 2}$ & 625423 & 624808 & 624574 & 624516 & 625298 & 624078 & 623933 & 623869 & \\
\hline 16 & $3 s^{2} 3 p^{2}\left({ }_{2}^{1} D\right)^{1} D 3 d^{2} F_{5 / 2}$ & 635007 & 634165 & 634000 & 633960 & 634513 & 633342 & 633251 & 633259 & \\
\hline 17 & $3 s^{2} 3 p^{2}\left({ }_{2}^{3} P\right)^{3} P 3 d^{4} F_{7 / 2}$ & 634942 & 634357 & 634228 & 634199 & 634500 & 633563 & 633535 & 633562 & \\
\hline 18 & $3 s^{2} 3 p^{2}\left({ }_{2}^{1} D\right)^{1} D 3 d^{2} F_{7 / 2}$ & 648315 & 647561 & 647406 & 647370 & 647867 & 646757 & 646697 & 646714 & \\
\hline 19 & $3 s^{2} 3 p^{2}\left({ }_{2}^{3} P\right)^{3} P 3 d^{4} D_{1 / 2}$ & 651015 & 650457 & 650308 & 650274 & 650426 & 649425 & 649391 & 649405 & \\
\hline 20 & $\left.3 s^{2} 3 p^{2}{ }_{2}^{3} P\right)^{3} P 3 d^{4} D_{3 / 2}$ & 651165 & 650565 & 650426 & 650395 & 650534 & 649508 & 649482 & 649508 & \\
\hline 21 & $\left.3 s^{2} 3 p^{2}{ }_{2}^{3} P\right)^{3} P 3 d^{4} F_{9 / 2}$ & 653961 & 653285 & 653147 & 653116 & 653581 & 652562 & 652523 & 652548 & \\
\hline 22 & $3 s^{2} 3 p^{2}\left({ }_{2}^{3} P\right)^{3} P 3 d^{4} D_{5 / 2}$ & 664563 & 663885 & 663743 & 663711 & 664015 & 662961 & 662923 & 662947 & \\
\hline 23 & $3 s^{2} 3 p^{2}\left({ }_{2}^{3} P\right)^{3} P 3 d^{4} D_{7 / 2}$ & 693413 & 692650 & 692498 & 692463 & 693051 & 691935 & 691887 & 691910 & \\
\hline 24 & $3 s^{2} 3 p^{2}\left({ }_{2}^{1} D\right)^{1} D 3 d^{2} G_{7 / 2}$ & 722577 & 720788 & 720541 & 720474 & 722174 & 719941 & 719726 & 719700 & \\
\hline 25 & $3 s^{2} S 3 p^{4}\left({ }_{2}^{3} P\right)^{2} P_{3 / 2}$ & 726764 & 725303 & 725038 & 724974 & 723123 & 721309 & 721031 & 720954 & \\
\hline 26 & $3 s^{2} 3 p^{2}\left({ }_{2}^{3} P\right)^{3} P 3 d^{4} P_{5 / 2}$ & 735073 & 733309 & 733065 & 732999 & 734228 & 732599 & 732370 & 732349 & \\
\hline 27 & $3 s^{2} 3 p^{2}\left({ }_{2}^{1} D\right)^{1} D 3 d^{2} G_{9 / 2}$ & 737751 & 736318 & 736149 & 736112 & 734773 & 732581 & 732383 & 732363 & 731502 \\
\hline 28 & $3 s^{2} 3 p^{2}\left({ }_{2}^{1} D\right)^{1} D 3 d^{2} P_{3 / 2}$ & 746899 & 745310 & 745105 & 745057 & 743652 & 741845 & 741558 & 741520 & 740598 \\
\hline 29 & $3 s^{2} 3 p^{2}\left({ }_{2}^{3} P\right)^{3} P 3 d^{4} P_{1 / 2}$ & 751364 & 749824 & 749626 & 749578 & 747946 & 746162 & 745891 & 745851 & \\
\hline 30 & $3 s^{2} 3 p^{2}\left({ }_{0}^{1} S\right)^{3} P 3 d^{2} D_{3 / 2}$ & 762822 & 760841 & 760510 & 760429 & 761590 & 759254 & 758834 & 758765 & \\
\hline 31 & $3 s^{2} S 3 p^{4}\left({ }_{2}^{3} P\right)^{2} P_{1 / 2}$ & 767755 & 766378 & 766151 & 766096 & 764162 & 762466 & 762226 & 762169 & \\
\hline 32 & $3 s^{2} 3 p^{2}\left({ }_{2}^{1} D\right)^{1} D 3 d^{2} D_{5 / 2}$ & 789398 & 787466 & 787171 & 787102 & 882747 & 879772 & 784118 & 784043 & \\
\hline 33 & $3 s^{2} 3 p^{2}\left({ }_{2}^{1} D\right)^{1} D 3 d^{2} D_{3 / 2}$ & 806669 & 804505 & 804227 & 804158 & 803380 & 800873 & 800493 & 800423 & \\
\hline 34 & $3 s^{2} 3 p^{2}\left({ }_{0}^{1} S\right)^{1} S 3 d^{2} D_{5 / 2}$ & 811857 & 809558 & 809212 & 809118 & 810796 & 808043 & 807649 & 807584 & 805800 \\
\hline 35 & $3 s^{2} 3 p^{2}\left({ }_{2}^{1} D\right)^{1} D 3 d^{2} P_{1 / 2}$ & 820141 & 817458 & 817109 & 817014 & 817286 & 814317 & 813735 & 813621 & \\
\hline 36 & $3 s^{2} 3 p^{2}\left({ }_{2}^{3} P\right)^{3} P 3 d^{2} F_{5 / 2}$ & 825926 & 823114 & 822808 & 822734 & 822379 & 819389 & 818900 & 818823 & \\
\hline 37 & $3 s^{2} 3 p^{2}\left({ }_{2}^{3} P\right)^{3} P 3 d^{2} F_{7 / 2}$ & 843870 & 840956 & 840675 & 840606 & 840276 & 837223 & 836771 & 836707 & 834392 \\
\hline 38 & $3 s^{2} 3 p^{2}\left({ }_{2}^{3} P\right)^{3} P 3 d^{4} P_{3 / 2}$ & 847064 & 844445 & 844106 & 844017 & 844148 & 841263 & 840691 & 840581 & 840300 \\
\hline 39 & $3 s^{2} 3 p^{2}\left({ }_{2}^{1} D\right)^{1} D 3 d^{2} S_{1 / 2}$ & 851999 & 849593 & 849255 & 849168 & 848953 & 846238 & 845796 & 845711 & \\
\hline 40 & $3 s^{2} 3 p^{2}\left({ }_{2}^{1} D\right)^{1} D 3 d^{2} D_{3 / 2}$ & 884728 & 881993 & 881632 & 881541 & 881735 & 878764 & 878211 & 878116 & \\
\hline 41 & $3 s^{2} 3 p^{2}\left({ }_{2}^{3} P\right)^{3} P 3 d^{2} D_{5 / 2}$ & 886080 & 883279 & 882939 & 882857 & 786707 & 784524 & 879239 & 879152 & 876810 \\
\hline
\end{tabular}

The corrections due to the Breit interaction and QED to the excited levels of Ge(XVIII) are shown in Fig. 3. Self-energy and vacuum polarization are the two major components in the QED correction [21]. As can be seen, the contribution of the Breit interaction is about $0.03 \%-0.99 \%$, and the contribution of QED is $-0.30 \%-0.07 \%$. The excited energy levels of Ge(XVIII) are all reduced by the mean value $0.18 \%$ due to the inclusion of the Breit interaction and QED corrections.
The data from the MCDF calculations with the Breit and QED effects for 39 levels are obtained from Fritzsche et al. [13], and energies from quasirelativistic HartreeFock and configuration interactions given by Applicable Data of Many-electron Atom energies and Transitions (ADAMANT) [31] are compared with the lowest 41 levels of Ge(XVIII) in Fig. 4. The present results in Fig. 4 are $\mathrm{VV}$ and $\mathrm{CV}$ calculations with $n=7$. For $3 s^{2} 3 p^{3}$, the VV results agree well with NIST in the range 


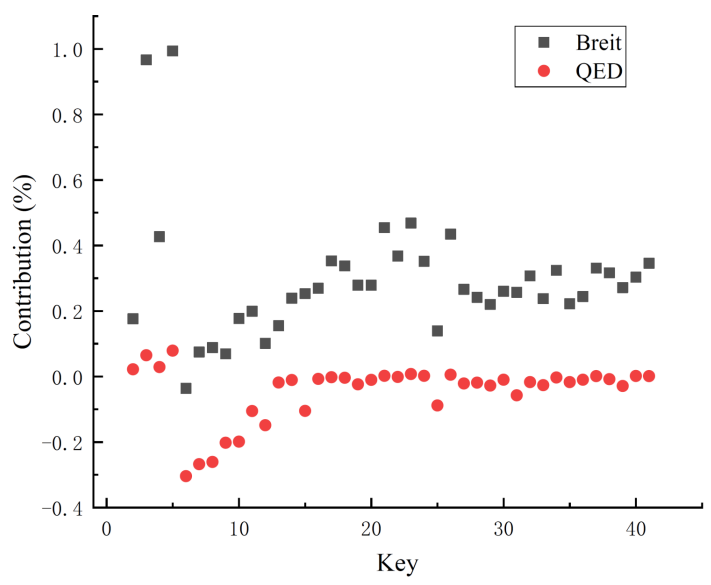

Fig. 3. The effects of the Breit interaction and QED corrections on the excitation energies of the Ge(XVIII) configurations obtained from the present MCDHF calculations.

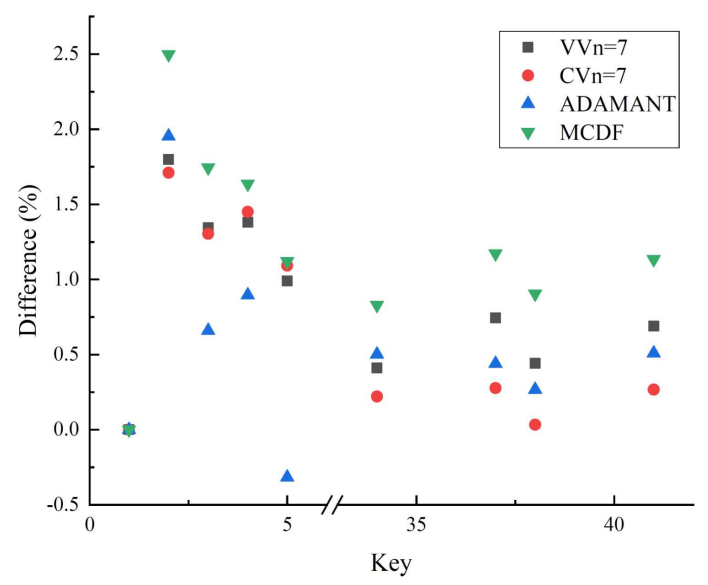

Fig. 4. Differences (in \%) of various theoretical energies from the NIST compiled values in Ge(XVIII).

of $0.99 \%$ to $1.80 \%$, and in the range of $1.03 \%$ to $1.71 \%$ for $\mathrm{CV}$. For $3 s^{2} 3 p^{2} 3 d$, the $\mathrm{VV}$ results agree well with NIST in the range of $0.41 \%$ to $0.74 \%$, and in the range of $0.03 \%$ to $0.27 \%$ for CV. The results from ADAMANT are in general agreement with NIST, but the difference of $3 s^{2} 3 p^{3}\left({ }_{1}^{2} P\right)^{2} P_{3 / 2}$ is $-0.32 \%$, which was dubious. This is because all the theoretical results were estimated. Theoretical results from Fritzsche et al. [13] differ the NIST in the range of $1.12 \%$ to $2.50 \%$ for $3 s^{2} 3 p^{3}$ and $0.91 \%$ to $1.17 \%$ for $3 s^{2} 3 p^{2} 3 d$, where valence correlation were not included in the calculation.

The Dirac-Fock wave functions with a minimum number of radial functions are not sufficient to represent the occupied orbitals. Extra configurations have to be added to adequately represent electron correlations (i.e. mixing coefficients). These extra configurations are represented by CSFs and must have the same angular momentum and parity as the occupied orbitals [32]. For instance, the level $3 s^{2} 3 p^{3}\left({ }_{3}^{4} S\right)^{4} S_{3 / 2}$ is represented by 0.91 $3 s^{2} 3 p^{3}\left({ }_{3}^{4} S\right)^{4} S_{3 / 2}$ and $0.333 s^{2} 3 p^{3}\left({ }_{1}^{2} P\right)^{2} P_{3 / 2}$.
The former two mixing coefficients for the wave functions of calculated levels are shown in Table II. The most important contributions to the total wave function of a given level are those from the same configuration. For example, the configuration-mixed wave function for the $3 s^{2} 3 p^{3}\left({ }_{3}^{4} S\right)^{4} S_{3 / 2}$ level is represented as $3 s^{2} 3 p^{3}\left({ }_{3}^{4} S\right)^{4} S_{3 / 2}=0.843 s^{2} 3 p^{3}\left({ }_{3}^{4} S\right)^{4} S_{3 / 2}+$ $0.113 s^{2} 3 p^{3}\left({ }_{1}^{2} P\right)^{2} P_{3 / 2}$, where 0.84 and 0.11 are contributions. Clearly, the present and the previous results are very close to one another in the description of the configuration-interaction wave functions. Because of the strong mixing, levels 12 and 15, levels 18 and 23, levels 28 , and 38 , and levels 32 and 41 have the same quantum labels in original output. With the help of JJ2LSJ [28], the levels mentioned above had been adjusted.

It should be noted that there is still around 1\% difference between calculated and experimental energies. The difference comes mainly from two parts. One is accuracy of experimental results. In this case, all levels of the ground configuration were determined with the M1 transitions observed by Denne et al. [33]. Sugar et al., determined that the ground configuration intervals relies on the smoothly varying predictions of energylevel values as a function of atomic number $Z$ provided by the codes from Desclaux and Grant et al., or Cowan code [34]. When NIST complied the values, these two papers were included in NIST Atomic Spectra Database Levels Form. However, the predictions of transition array were not adopted in NIST Atomic Spectra Database Lines Form. In a sense, the predictions for levels and transitions are still to be checked again. Another is the method of calculations. That is to say, the number of reference configuration and reference orbitals is important in calculation. Due to the limitation of computational resources and GRASP2K, principal number and the angular momentum quantum number was limited in our calculation. From Table II, we can find the contribution from present calculation is slightly different with NIST. Therefore, more configurations may be tested for more accurate results in the future.

The lifetime for level $j$ is defined as $\tau=1 / \sum_{j} A_{j i}$. Lifetime is a measurable quantity, and it can be a good check on the accuracy of this calculation. Therefore, lifetimes for the present 36 levels in Ge(XVIII) in length and velocity are listed in Table III, which include the contributions from all possible E1 and M2 radiative decays from level $j$. Other theoretical investigations from Fritzsche et al. [13] and Vilkas and Ishikawa [15] are presented in Table III. The present lifetimes are almost dominated by the E1 transitions. The present results are consistent with results obtained by Fritzsche et al. [13]. The value $\tau_{v} / \tau_{l}$ for present calculation is in the range of 0.90 to 1.05 , while 0.94 to 1.14 for Fritzsche et al. [13]. The data from Vilkas and Ishikawa [15] agree well with most levels, but results for levels 36-38 and 40-41 are sharp distinction. More precise measurement is needed to assess the accuracy of these theoretical results. 
The configuration mixing coefficients and contributions for the lowest levels in Ge(XVIII).

TABLE II

\begin{tabular}{|c|c|c|c|c|}
\hline \multirow{2}{*}{ Key } & \multirow{2}{*}{ Configuration } & \multirow{2}{*}{ Mix } & \multicolumn{2}{|c|}{ Contribution } \\
\hline & & & Present & NIST [30] \\
\hline 1 & $3 s^{2} 3 p^{3}\left({ }_{3}^{4} S\right)^{4} S_{3 / 2}$ & $0.91(1)+0.33(4)$ & $0.83(1)+0.11(5)$ & $0.86(1)+0.11(5)$ \\
\hline 2 & $3 s^{2} 3 p^{3}\left({ }_{3}^{2} D\right)^{2} D_{3 / 2}$ & $0.85(2)-0.39(4)$ & $0.72(2)+0.15(5)$ & $0.75(2)+0.16(5)$ \\
\hline 3 & $3 s^{2} 3 p^{3}\left({ }_{3}^{2} D\right)^{2} D_{5 / 2}$ & $0.99(3)$ & $0.97(3)$ & $1.00(3)$ \\
\hline 4 & $3 s^{2} 3 p^{3}\left({ }_{1}^{2} P\right)^{2} P_{1 / 2}$ & $0.98(4)$ & $0.97(4)$ & $1.00(4)$ \\
\hline 5 & $3 s^{2} 3 p^{3}\left({ }_{1}^{2} P\right)^{2} P_{3 / 2}$ & $0.84(5)+0.46(2)$ & $0.70(5)+0.22(2)$ & $0.73(5)+0.22(2)$ \\
\hline 6 & $3 s^{2} S 3 p^{4}\left({ }_{2}^{3} P\right)^{4} P_{5 / 2}$ & $0.92(6)+0.29(26)$ & $0.85(6)+0.09(26)$ & \\
\hline 7 & $3 s^{2} S 3 p^{4}\left({ }_{2}^{3} P\right)^{4} P_{3 / 2}$ & $-0.92(7)-0.31(28)$ & $0.85(7)+0.10(28)$ & \\
\hline 8 & $3 s^{2} S 3 p^{4}\left({ }_{2}^{3} P\right)^{4} P_{1 / 2}$ & $0.91(7)+0.31(29)$ & $0.83(7)+0.10(29)$ & \\
\hline 9 & $3 s^{2} S 3 p^{4}\left({ }_{2}^{1} D\right)^{2} D_{3 / 2}$ & $0.82(9)-0.39(33)$ & $0.68(9)+0.15(33)$ & \\
\hline 10 & $3 s^{2} S 3 p^{4}\left({ }_{2}^{1} D\right)^{2} D_{5 / 2}$ & $-0.86(10)+0.41(41)$ & $0.73(10)+0.17(41)$ & \\
\hline 11 & $3 s^{2} 3 p^{2}\left({ }_{2}^{3} P\right)^{3} P 3 d^{2} P_{3 / 2}$ & $-0.66(11)+0.61(7)$ & $0.43(11)+0.38(7)$ & \\
\hline 12 & $3 s^{2} 3 p^{2}\left({ }_{2}^{3} P\right)^{3} P 3 d^{2} P_{1 / 2}$ & $-0.47(12)+0.58(15)$ & $0.23(12)+0.34(15)$ & \\
\hline 13 & $3 s^{2} 3 p^{2}\left({ }_{2}^{3} P\right)^{3} P 3 d^{4} F_{3 / 2}$ & $0.92(13)+0.24(20)$ & $0.85(13)+0.06(20)$ & \\
\hline 14 & $3 s^{2} 3 p^{2}\left({ }_{2}^{3} P\right)^{3} P 3 d^{4} F_{5 / 2}$ & $0.88(14)+0.38(22)$ & $0.78(14)+0.15(22)$ & \\
\hline 15 & $3 s^{2} S 3 p^{4}\left({ }_{0}^{1} S\right)^{2} S_{1 / 2}$ & $0.63(15)+0.47(12)$ & $0.40(15)+0.22(12)$ & \\
\hline 16 & $3 s^{2} 3 p^{2}\left({ }_{2}^{1} D\right)^{1} D 3 d^{2} F_{5 / 2}$ & $-0.66(16)-0.55(36)$ & $0.43(16)+0.30(36)$ & \\
\hline 17 & $\left.3 s^{2} 3 p^{2}{ }_{2}^{3} P\right)^{3} P 3 d^{4} F_{7 / 2}$ & $0.93(17)+0.29(23)$ & $0.87(17)+0.09(23)$ & \\
\hline 18 & $3 s^{2} 3 p^{2}\left({ }_{2}^{1} D\right)^{1} D 3 d^{2} F_{7 / 2}$ & $0.58(18)-0.63(23)$ & $0.33(18)+0.40(23)$ & \\
\hline 19 & $\left.3 s^{2} 3 p^{2}{ }_{2}^{3} P\right)^{3} P 3 d^{4} D_{1 / 2}$ & $-0.90(19)-0.29(31)$ & $0.81(19)+0.08(31)$ & \\
\hline 20 & $\left.3 s^{2} 3 p^{2}{ }_{2}^{3} P\right)^{3} P 3 d^{4} D_{3 / 2}$ & $0.92(20)-0.28(13)$ & $0.84(20)+0.08(13)$ & \\
\hline 21 & $\left.3 s^{2} 3 p^{2}{ }_{2}^{3} P\right)^{3} P 3 d^{4} F_{9 / 2}$ & $0.94(21)-0.31(27)$ & $0.88(21)+0.10(27)$ & \\
\hline 22 & $\left.3 s^{2} 3 p^{2}{ }_{2}^{3} P\right)^{3} P 3 d^{4} D_{5 / 2}$ & $0.78(22)+0.34(16)$ & $0.61(22)+0.12(16)$ & \\
\hline 23 & $\left.3 s^{2} 3 p^{2}{ }_{2}^{3} P\right)^{3} P 3 d^{4} D_{7 / 2}$ & $0.68(23)+0.42(18)$ & $0.46(23)+0.18(18)$ & \\
\hline 24 & $3 s^{2} 3 p^{2}\left({ }_{2}^{1} D\right)^{1} D 3 d^{2} G_{7 / 2}$ & $-0.91(24)-0.33(18)$ & $0.84(24)+0.11(18)$ & \\
\hline 25 & $3 s^{2} S 3 p^{4}\left({ }_{2}^{3} P\right)^{2} P_{3 / 2}$ & $0.65(25)+0.48(11)$ & $0.42(25)+0.23(11)$ & \\
\hline 26 & $3 s^{2} 3 p^{2}\left({ }_{2}^{3} P\right)^{3} P 3 d^{4} P_{5 / 2}$ & $0.88(26)-0.26(6)$ & $0.77(26)+0.07(6)$ & \\
\hline 27 & $3 s^{2} 3 p^{2}\left({ }_{2}^{1} D\right)^{1} D 3 d^{2} G_{9 / 2}$ & $0.94(27)+0.31(21)$ & $0.88(27)+0.10(21)$ & $0.80(27)+0.07(21)$ \\
\hline 28 & $3 s^{2} 3 p^{2}\left({ }_{2}^{1} D\right)^{1} D 3 d^{2} P_{3 / 2}$ & $0.81(28)+0.31(38)$ & $0.53(28)+0.25(11)$ & $0.56(28)+0.15(11)$ \\
\hline 29 & $\left.3 s^{2} 3 p^{2}{ }_{2}^{3} P\right)^{3} P 3 d^{4} P_{1 / 2}$ & $-0.77(29)-0.37(35)$ & $0.60(29)+0.14(35)$ & \\
\hline 30 & $3 s^{2} 3 p^{2}\left({ }_{0}^{1} S\right)^{3} P 3 d^{2} D_{3 / 2}$ & $0.58(30)+0.58(40)$ & $0.33(30)+0.33(40)$ & \\
\hline 31 & $3 s^{2} S 3 p^{4}\left({ }_{2}^{3} P\right)^{2} P_{1 / 2}$ & $-0.57(31)-0.45(12)$ & $0.33(31)+0.20(12)$ & \\
\hline 32 & $3 s^{2} 3 p^{2}\left({ }_{2}^{1} D\right)^{1} D 3 d^{2} D_{5 / 2}$ & $0.64(32)-0.47(41)$ & $0.41(32)+0.05(41)$ & \\
\hline 33 & $3 s^{2} 3 p^{2}\left({ }_{2}^{1} D\right)^{1} D 3 d^{2} D_{3 / 2}$ & $0.84(33)+0.38(9)$ & $0.71(33)+0.15(9)$ & \\
\hline 34 & $3 s^{2} 3 p^{2}\left({ }_{0}^{1} S\right)^{1} S 3 d^{2} D_{5 / 2}$ & $0.72(34)+0.53(41)$ & $0.52(34)+0.28(41)$ & $0.51(34)+0.08(41)$ \\
\hline 35 & $3 s^{2} 3 p^{2}\left({ }_{2}^{1} D\right)^{1} D 3 d^{2} P_{1 / 2}$ & $-0.76(35)-0.45(12)$ & $0.57(35)+0.20(12)$ & \\
\hline 36 & $3 s^{2} 3 p^{2}\left({ }_{2}^{3} P\right)^{3} P 3 d^{2} F_{5 / 2}$ & $0.57(36)-0.49(16)$ & $0.33(36)+0.25(16)$ & \\
\hline 37 & $3 s^{2} 3 p^{2}\left({ }_{2}^{3} P\right)^{3} P 3 d^{2} F_{7 / 2}$ & $0.78(37)-0.58(18)$ & $0.61(37)+0.34(18)$ & $0.40(37)+0.34(18)$ \\
\hline 38 & $3 s^{2} 3 p^{2}\left({ }_{2}^{3} P\right)^{3} P 3 d^{4} P_{3 / 2}$ & $0.73(38)+0.50(11)$ & $0.65(38)+0.09(38)$ & $0.64(38)+0.27(38)$ \\
\hline 39 & $3 s^{2} 3 p^{2}\left({ }_{2}^{1} D\right)^{1} D 3 d^{2} S_{1 / 2}$ & $0.78(39)+0.37(15)$ & $0.62(39)+0.14(15)$ & \\
\hline 40 & $3 s^{2} 3 p^{2}\left({ }_{2}^{1} D\right)^{1} D 3 d^{2} D_{3 / 2}$ & $-0.74(40)+0.62(30)$ & $0.54(40)+0.38(30)$ & \\
\hline 41 & $\left.3 s^{2} 3 p^{2}{ }_{2}^{3} P\right)^{3} P 3 d^{2} D_{5 / 2}$ & $0.22(41)+0.64(32)$ & $0.41(41)+0.23(32)$ & $0.39(41)+0.23(32)$ \\
\hline
\end{tabular}

The transition rate, the weighted oscillator strength, and the line strength were given in the Coulomb (velocity) and the Babushkin (length) gauges in this calculation. Also, for the electric transitions the relative difference $\mathrm{d} T\left(\mathrm{~d} T=\operatorname{abs}\left(A_{l}-A_{v}\right) / \max \left(A_{l}, A_{v}\right)\right)$ between the transition rates in length and velocity gauges are given. A value close to $\mathrm{d} T=0$ for an allowed transition is a known accuracy indicator [35]. In many cases the values are reasonably close to 0 , as shown in Fig. 5. However, in other cases, for example, the difference of transition $3 s^{2} 3 p^{2}\left({ }_{2}^{1} D\right)^{1} D 3 d^{2} F_{5 / 2}-3 s^{2} 3 p^{3}\left({ }_{3}^{2} D\right)^{2} D_{3 / 2}$ can be larger than 0.9. In particular, these calculations present provide comprehensive new data for E2, M1, and M2 transitions for Ge(XVIII), for which there is no existent data for public. This will help with the identification of spectral lines of $\mathrm{Ge}(\mathrm{XVIII})$. Owing to the space limitations, 
Calculated lifetimes $\left(\mathrm{s}^{-1}\right)$ of the lowest 36 excited even- and odd-parity states in Ge(XVIII). $a(b)=a \times 10^{b}$. TABLE III

\begin{tabular}{|c|c|c|c|c|c|c|}
\hline \multirow{2}{*}{ Key } & \multirow{2}{*}{ Configuration } & \multicolumn{2}{|c|}{ MCDHF } & \multicolumn{2}{|c|}{ MCDF } & \multirow{2}{*}{ MR-MP } \\
\hline & & $\tau_{l}$ & $\tau_{v}$ & $\tau_{l}$ & $\tau_{v}$ & \\
\hline 6 & $3 s^{2} S 3 p^{4}\left({ }_{2}^{3} P\right)^{4} P_{5 / 2}$ & $3.15(-10)$ & $2.96(-10)$ & $3.19(-10)$ & $3.12(-10)$ & $2.38(-10)$ \\
\hline 7 & $3 s^{2} S 3 p^{4}\left({ }_{2}^{3} P\right)^{4} P_{3 / 2}$ & $2.58(-10)$ & $2.46(-10)$ & $2.64(-10)$ & $2.61(-10)$ & $2.81(-10)$ \\
\hline 8 & $3 s^{2} S 3 p^{4}\left({ }_{2}^{3} P\right)^{4} P_{1 / 2}$ & $2.32(-10)$ & $2.21(-10)$ & $2.36(-10)$ & $2.32(-10)$ & $2.49(-10)$ \\
\hline 9 & $3 s^{2} S 3 p^{4}\left({ }_{2}^{1} D\right)^{2} D_{3 / 2}$ & $1.29(-10)$ & $1.24(-10)$ & $1.32(-10)$ & $1.31(-10)$ & $1.39(-10)$ \\
\hline 10 & $3 s^{2} S 3 p^{4}\left({ }_{2}^{1} D\right)^{2} D_{5 / 2}$ & $1.61(-10)$ & $1.55(-10)$ & $1.65(-10)$ & $1.62(-10)$ & $1.72(-10)$ \\
\hline 11 & $3 s^{2} 3 p^{2}\left({ }_{2}^{3} P\right)^{3} P 3 d^{2} P_{3 / 2}$ & $7.15(-11)$ & $6.99(-11)$ & $7.40(-11)$ & $7.18(-11)$ & $7.75(-11)$ \\
\hline 12 & $\left.3 s^{2} 3 p^{2}{ }_{2}^{3} P\right)^{3} P 3 d^{2} P_{1 / 2}$ & $5.04(-11)$ & $4.89(-11)$ & $5.12(-11)$ & $4.88(-11)$ & $5.37(-11)$ \\
\hline 13 & $\left.3 s^{2} 3 p^{2}{ }_{2}^{3} P\right)^{3} P 3 d^{4} F_{3 / 2}$ & $4.60(-10)$ & $4.53(-10)$ & $4.87(-10)$ & $4.60(-10)$ & $5.18(-10)$ \\
\hline 14 & $\left.3 s^{2} 3 p^{2}{ }_{2}^{3} P\right)^{3} P 3 d^{4} F_{5 / 2}$ & $8.60(-10)$ & $8.99(-10)$ & $9.15(-10)$ & $8.66(-10)$ & $9.85(-10)$ \\
\hline 15 & $3 s^{2} S 3 p^{4}\left({ }_{0}^{1} S\right)^{2} S_{1 / 2}$ & $9.05(-11)$ & $8.73(-11)$ & $9.08(-11)$ & $8.58(-11)$ & $9.45(-11)$ \\
\hline 16 & $3 s^{2} 3 p^{2}\left({ }_{2}^{1} D\right)^{1} D 3 d^{2} F_{5 / 2}$ & $1.62(-09)$ & $1.69(-09)$ & $1.71(-09)$ & $1.63(-09)$ & $1.75(-09)$ \\
\hline 17 & $3 s^{2} 3 p^{2}\left({ }_{2}^{3} P\right)^{3} P 3 d^{4} F_{7 / 2}$ & $9.13(-09)$ & $9.94(-09)$ & $9.46(-09)$ & $9.24(-09)$ & $9.89(-09)$ \\
\hline 18 & $3 s^{2} 3 p^{2}\left({ }_{2}^{1} D\right)^{1} D 3 d^{2} F_{7 / 2}$ & $8.99(-09)$ & $9.58(-09)$ & $8.30(-09)$ & $7.37(-09)$ & $7.51(-09)$ \\
\hline 19 & $\left.3 s^{2} 3 p^{2}{ }_{2}^{3} P\right)^{3} P 3 d^{4} D_{1 / 2}$ & $1.53(-10)$ & $1.51(-10)$ & $1.58(-10)$ & $1.51(-10)$ & $1.64(-10)$ \\
\hline 20 & $3 s^{2} 3 p^{2}\left({ }_{2}^{3} P\right)^{3} P 3 d^{4} D_{3 / 2}$ & $1.99(-10)$ & $2.02(-10)$ & $2.15(-10)$ & $2.06(-10)$ & $2.28(-10)$ \\
\hline 21 & $3 s^{2} 3 p^{2}\left({ }_{2}^{3} P\right)^{3} P 3 d^{4} F_{9 / 2}$ & $7.37(-03)$ & $7.37(-03)$ & & & \\
\hline 22 & $3 s^{2} 3 p^{2}\left({ }_{2}^{3} P\right)^{3} P 3 d^{4} D_{5 / 2}$ & $2.78(-10)$ & $2.86(-10)$ & $2.98(-10)$ & $2.85(-10)$ & $3.34(-10)$ \\
\hline 23 & $3 s^{2} 3 p^{2}\left({ }_{2}^{3} P\right)^{3} P 3 d^{4} D_{7 / 2}$ & $5.24(-09)$ & $5.80(-09)$ & $6.84(-09)$ & $7.09(-09)$ & $9.52(-09)$ \\
\hline 24 & $3 s^{2} 3 p^{2}\left({ }_{2}^{1} D\right)^{1} D 3 d^{2} G_{7 / 2}$ & $5.16(-10)$ & $5.44(-10)$ & $5.34(-10)$ & $5.08(-10)$ & $5.80(-10)$ \\
\hline 25 & $3 s^{2} S 3 p^{4}\left({ }_{2}^{3} P\right)^{2} P_{3 / 2}$ & $8.99(-12)$ & $9.12(-12)$ & $8.62(-12)$ & $8.21(-12)$ & $9.14(-12)$ \\
\hline 26 & $3 s^{2} 3 p^{2}\left({ }_{2}^{3} P\right)^{3} P 3 d^{4} P_{5 / 2}$ & $7.98(-12)$ & $8.20(-12)$ & $7.63(-12)$ & $7.24(-12)$ & $8.10(-12)$ \\
\hline 27 & $3 s^{2} 3 p^{2}\left({ }_{2}^{1} D\right)^{1} D 3 d^{2} G_{9 / 2}$ & $3.56(-01)$ & $3.56(-01)$ & & & \\
\hline 28 & $3 s^{2} 3 p^{2}\left({ }_{2}^{1} D\right)^{1} D 3 d^{2} P_{3 / 2}$ & $8.35(-12)$ & $8.52(-12)$ & $8.20(-10)$ & $7.79(-10)$ & $8.60(-12)$ \\
\hline 29 & $3 s^{2} 3 p^{2}\left({ }_{2}^{3} P\right)^{3} P 3 d^{4} P_{1 / 2}$ & $7.87(-12)$ & $8.03(-12)$ & $7.52(-12)$ & $7.18(-12)$ & $8.00(-12)$ \\
\hline 30 & $3 s^{2} 3 p^{2}\left({ }_{0}^{1} S\right)^{3} P 3 d^{2} D_{3 / 2}$ & $2.71(-11)$ & $2.74(-11)$ & $2.39(-11)$ & $2.27(-11)$ & $2.70(-11)$ \\
\hline 31 & $3 s^{2} S 3 p^{4}\left({ }_{2}^{3} P\right)^{2} P_{1 / 2}$ & $8.02(-12)$ & $8.15(-12)$ & $7.66(-12)$ & $7.32(-12)$ & $8.00(-12)$ \\
\hline 32 & $3 s^{2} 3 p^{2}\left({ }_{2}^{1} D\right)^{1} D 3 d^{2} D_{5 / 2}$ & $1.05(-11)$ & $1.07(-11)$ & $1.06(-11)$ & $1.01(-11)$ & $1.12(-11)$ \\
\hline 33 & $3 s^{2} 3 p^{2}\left({ }_{2}^{1} D\right)^{1} D 3 d^{2} D_{3 / 2}$ & $8.08(-12)$ & $8.31(-12)$ & $8.93(-12)$ & $8.51(-12)$ & $8.00(-12)$ \\
\hline 34 & $3 s^{2} 3 p^{2}\left({ }_{0}^{1} S\right)^{1} S 3 d^{2} D_{5 / 2}$ & $2.56(-11)$ & $2.72(-11)$ & $2.42(-11)$ & $2.36(-11)$ & $2.70(-11)$ \\
\hline 35 & $3 s^{2} 3 p^{2}\left({ }_{2}^{1} D\right)^{1} D 3 d^{2} P_{1 / 2}$ & $1.00(-11)$ & $1.01(-11)$ & $9.55(-12)$ & $9.13(-12)$ & $8.00(-12)$ \\
\hline 36 & $3 s^{2} 3 p^{2}\left({ }_{2}^{3} P\right)^{3} P 3 d^{2} F_{5 / 2}$ & $6.95(-12)$ & $7.26(-12)$ & $6.51(-12)$ & $6.26(-12)$ & $6.80(-11)$ \\
\hline 37 & $3 s^{2} 3 p^{2}\left({ }_{2}^{3} P\right)^{3} P 3 d^{2} F_{7 / 2}$ & $6.56(-12)$ & $6.89(-12)$ & $6.34(-12)$ & $6.11(-12)$ & $6.70(-11)$ \\
\hline 38 & $3 s^{2} 3 p^{2}\left({ }_{2}^{3} P\right)^{3} P 3 d^{4} P_{3 / 2}$ & $9.37(-12)$ & $9.52(-12)$ & $8.93(-12)$ & $8.51(-12)$ & $9.10(-12)$ \\
\hline 39 & $3 s^{2} 3 p^{2}\left({ }_{2}^{1} D\right)^{1} D 3 d^{2} S_{1 / 2}$ & $8.83(-12)$ & $9.13(-12)$ & $8.43(-12)$ & $8.21(-12)$ & $9.00(-11)$ \\
\hline 40 & $3 s^{2} 3 p^{2}\left({ }_{2}^{1} D\right)^{1} D 3 d^{2} D_{3 / 2}$ & $7.71(-12)$ & $8.05(-12)$ & $7.42(-12)$ & $7.90(-12)$ & $7.90(-10)$ \\
\hline 41 & $3 s^{2} 3 p^{2}\left({ }_{2}^{3} P\right)^{3} P 3 d^{2} D_{5 / 2}$ & $7.30(-12)$ & $7.64(-12)$ & $7.05(-12)$ & $6.80(-12)$ & $7.80(-11)$ \\
\hline
\end{tabular}

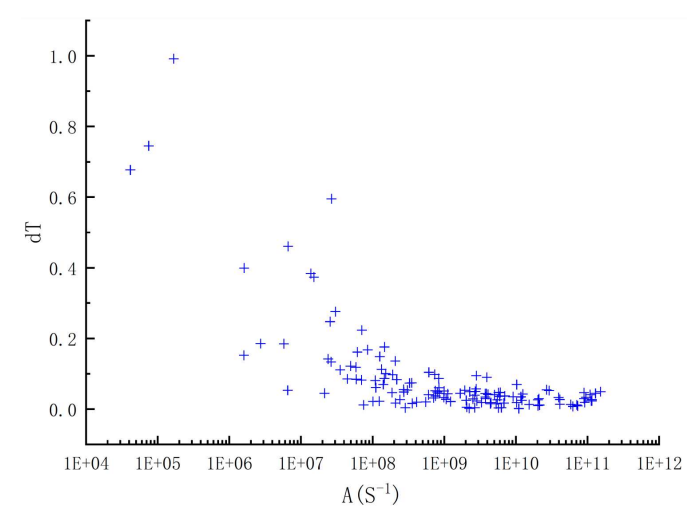

Fig. 5. Scatterplot of $\mathrm{d} T$ and $A\left[\mathrm{~S}^{-1}\right]$ for all $\mathrm{E} 1$ transitions. full tables of E1, E2, M1, and M2 transitions data will be provided as the supplemental material in conjunction with the E-mail.

\section{Conclusion}

MCDHF and RCI calculations for $3 s^{2} 3 p^{3}, 3 s 3 p^{4}$, and $3 s^{2} 3 p^{2} 3 d$ configurations of $\mathrm{P}$-like $\mathrm{Ge}$ are presented. Fine structure energy levels, oscillator strengths, and transition probabilities for E1, E2, M1, and M2 transitions among levels belonging to these levels are performed. The valence-valence and core-valence correlation effects are accounted for in a systematic way. The calculated energy levels and weighted oscillator strengths with corevalence correlation effect show good agreements with 
both theoretical and experimental data from the literature. The computed wavelengths are almost of spectroscopic accuracy, aiding line identification in spectra. Our results are useful for many applications such as controlled thermonuclear fusion, laser and plasma physics, as well as astrophysics.

\section{Acknowledgments}

This work was supported by the National Natural Science Foundation of China (Grant No. 11604284).

\section{References}

[1] W. Jarrah, D. Benerdjem, J.C. Pain, J. Duan, High Energy Dens. Phys. 24, 64 (2017).

[2] H. Shakeel, S. Arshad, S.U. Haq, A. Nadeem, Phys. Plasmas 23, 053504 (2016).

[3] M. Comet, J.C. Pain, F. Gilleron, et al., AIP Conf. Proc. 1811, 070001 (2017).

[4] E.G. Hill, G. Pérez-Callejo, S.J. Rose, High Energy Dens. Phys. 26, 56 (2018).

[5] F. Hu, C. Han, M.F. Mei, J.M. Yang, J.Y. Zhang, G. Jiang, Radiat. Eff. Def. Solids 170, 407 (2015).

[6] F. Hu, Y. Sun, M.F. Mei, C.C. Sang, J. Xuzhou Inst. Technol. Nat. Sci. Ed. 33, 37 (2018) (in Chinese).

[7] F. Hu, Y. Sun, M.F. Mei, C. Han, C.C. Sang, J. Xuzhou Inst. Technol. 34, 50 (2019).

[8] J. Sugar, V. Kaufman, J. Opt. Soc. Am. B 1, 218 (1984).

[9] K.N. Huang, At. Data Nucl. Data Tables 30, 313 (1984).

[10] E. Biémont, J.E. Hansen, Phys. Scr. 31, 509 (1985).

[11] C. Froese Fischer, M. Godefroid, J. Phys. B 19, 137 (1986).

[12] L.J. Curtis, Z.B. Rudzikas, D.G. Ellis, Phys. Rev. A 44, 776 (1991).

[13] S. Fritzsche, C. Froese Fischer, B. Fricke, At. Data Nucl. Data Tables 68, 149 (1998).

[14] E. Charro, I. Martín, M.A. Serna, J. Phys. B 33, 1753 (2000).

[15] M.J. Vilkas, Y. Ishikawa, J. Phys. B 37, 4763 (2004).

[16] E. Träbert, M. Grieser, C. Krantz, R. Repnow, A. Wolf, F.J. Diaz, Y. Ishikawa, J.A. Santana, J. Phys. B 45, 215003 (2012).
[17] E. Träbert, Phys. Scr. 89, 114003 (2014).

[18] E. Träbert, Atoms 2, 15 (2014).

[19] P. Jönsson, G. Gaigalas, J. Bieroń, C. Froese Fischer, I.P. Grant, Comput. Phys. Commun. 184, 2197 (2013).

[20] C. Froese Fischer, G. Gaigalas, P. Jönsson, J. Bieroń, Comput. Phys. Commun. 237, 184 (2019).

[21] F. Hu, J.M. Yang, C.K. Wang, L.F. Jing, S.B. Chen, G. Jiang, H. Liu, L.H. Hao, Phys. Rev. A 84, 042506 (2011).

[22] F. Hu, Y. Sun, M.F. Mei, C.C. Sang, J. Appl. Spectrosc. 85, 749 (2018).

[23] I.P. Grant, Relativistic Quantum Theory of Atoms, Molecules, Springer, New York 2007.

[24] B.J. McKenzie, I.P. Grant, P.H. Norrington, Comput. Phys. Commun. 21, 233 (1980).

[25] K.G. Dyall, I.P. Grant, C.T. Johnson, F.A. Parpia, E.P. Plummer, Comput. Phys. Commun. 55, 425 (1989).

[26] J. Olsen, G.M. Godefroid, P. Jönsson, Påke Malmqvist, C. Froese Fischer, Phys. Rev. E 52, 4499 (1995).

[27] L. Sturesson, P. Jönsson, C. Froese Fischer, Comput. Phys. Commun. 177, 539 (2007).

[28] G. Gaigalas, C. Froese Fischer, P. Rynkun, P. Jönsson, Atoms 5, 6 (2017).

[29] C. Nazé, E. Gaidamauskas, G. Gaigalas, M. Godefroid, P. Jönsson, Comput. Phys. Commun. 184, 2187 (2013).

[30] A. Kramida, Atomic Transition Probability Bibliographic Database (version 9.0), National Institute of Standards, Technology, Gaithersburg (MD) (2019).

[31] ADAMANT (Applicable Data of Many-Electron Atom Energies, Transitions).

[32] J. Gillaspy, Trapping Highly Charged Ions: Fundamentals, Applications, Nova Publisher, New York 2001.

[33] B. Denne, E. Hinnov, S. Suckewer, J. Timberlake, J. Opt. Soc. Am. B 1, 296 (1984).

[34] J. Sugar, V. Kaufman, W.L. Rowan, J. Opt. Soc. Am. B 8, 22 (1991).

[35] J. Ekman, M. Godefroid, H. Hartman, Atoms 2, 215 (2014). 